\title{
Contribution of sexual desire and motives to the compulsive use of cybersex
}

\author{
FARAH BEN BRAHIM $^{1}$, STEPHANE ROTHEN ${ }^{2,3}$, FRANCESCO BIANCHI-DEMICHELI ${ }^{3}$, ROBERT COURTOIS ${ }^{1,4}$ \\ and YASSER KHAZAAL ${ }^{5,6 *}$ \\ ${ }^{1}$ Department of Psychology, University of Tours, Tours, France \\ ${ }^{2}$ Research Center for Statistics, University of Geneva, Geneva, Switzerland \\ ${ }^{3}$ Department of Mental Health and Psychiatry, Geneva University Hospitals, Geneva, Switzerland \\ ${ }^{4}$ Psychiatric University Clinic, University Hospital of Tours (CHRU), Tours, France \\ ${ }^{5}$ Addiction Medicine, Department of Psychiatry, Lausanne University Hospital and Lausanne University, Lausanne, Switzerland \\ ${ }^{6}$ Research Centre, University Institute of Mental Health at Montreal, Montreal, Canada
}

(Received: November 13, 2018; revised manuscript received: March 9, 2019; second revised manuscript received: July 22, 2019; accepted: August 7, 2019)

\begin{abstract}
Background and aims: Cybersex is increasingly associated with concerns about compulsive use. The aim of this study was to assess the roles of motives and sexual desire in the compulsive use of cybersex. Methods: The sample consisted of 306 cybersex users (150 men and 156 women). The participants were assessed using the Compulsive Internet Use Scale (CIUS) adapted for cybersex, the Cybersex Motives Questionnaire (enhancement, coping, and social motives), and the Sexual Desire Inventory-2 (dyadic and solitary sexual desire). Results: For both genders, coping motive was associated with CIUS score. For women, an additional association with social motives was found whereas an association with sexual desire was found for men. Conclusion: The study showed gender differences in the contributors to sex-related CIUS scores.
\end{abstract}

Keywords: compulsive cybersex, Cybersex Motives Questionnaire, sexual desire, Internet addiction, cybersex addiction

\section{INTRODUCTION}

Cybersex includes a wide range of sex-related Internet activities, such as pornography, webcam, sex chat, online sex games, and dating (Döring, 2009; Wéry \& Billieux, 2017). In addition to virtual encounters, cybersex can facilitate meeting real romantic and sexual partners. Other positive impacts of cybersex have been noted (Grov, Gillespie, Royce, \& Lever, 2011). For instance, it can enhance sexual arousal and facilitate engagement in sexual practices (Allen, Kannis-Dymand, \& Katsikitis, 2017). It can also help couples with sexual stimulation or with the exploration of new sexual practices (Albright, 2008; Philaretou, Mahfouz, \& Allen, 2005).

Compulsive cybersex use seems to affect a small proportion of cybersex consumers (Dufour et al., 2016; Frangos, Frangos, \& Sotiropoulos, 2010; Kafka, 2010) and is possibly associated with psychosocial distress, disturbances in fulfilling daily life responsibilities, and sleeprelated problems (Grubbs, Volk, Exline, \& Pargament, 2015; Karila et al., 2014). In several studies involving males and females, compulsive cybersex users, compared to controls, showed greater arousal and cue reactivity from watching porn (Brand et al., 2011; Laier \& Brand, 2014; Laier, Pawlikowski, Pekal, Schulte, \& Brand, 2013; Laier, Pekal, \& Brand, 2014). However, despite the importance of the topic, it has been little studied
(Brand et al., 2011). In particular, little is known about the possible psychological determinants of compulsive cybersex use (Franc et al., 2018).

Negative emotions combined with negative urgency (the tendency to act impulsively in negative emotional situations) have been found to contribute to cybersex use (Wéry, Deleuze, Canale, \& Billieux, 2018), probably as a way to cope with negative affect. Motives, especially those related to coping (i.e., to escape from real-life problems), are known to influence substance use (Benschop et al., 2015), behavioral addictions (Billieux et al., 2011; Király et al., 2015; Zanetta Dauriat et al., 2011), and compulsive cybersex (Brand, Laier, \& Young, 2014). Cybersex, with or without masturbation (Putnam \& Maheu, 2000; Wéry, Karila, Sutter, \& Billieux, 2014), is used by some people as a coping strategy to manage negative emotions (Barrault, Hegbe, Bertsch, \& Courtois, 2016; Southern, 2008). Enhancement and social motives (Franc et al., 2018) may also play a role in compulsive cybersex. Studies on Internet dating have underlined the role of social motives (Sumter,

\footnotetext{
* Corresponding author: Yasser Khazaal; Addiction Medicine, Department of Psychiatry, Lausanne University Hospital and Lausanne University, Bugnon 23, Lausanne 1011, Switzerland; Phone: +41 2131484 00; Fax: +41 2131473 51; E-mail: yasser. khazaal@chuv.ch
}

This is an open-access article distributed under the terms of the Creative Commons Attribution-NonCommercial 4.0 International License, which permits unrestricted use, distribution, and reproduction in any medium for non-commercial purposes, provided the original author and source are credited, a link to the CC License is provided, and changes - if any - are indicated. 
Vandenbosch, \& Ligtenberg, 2017) and the expectation of arousal when watching porn (Young, 2008).

Sexual desire is a driving component of sexual fantasy life and activity (Levine, 2003; Pfaus, 2009). Moderate associations have been found between sexual desire and cybersex enhancement motives (Commission of the European Communities, 2002; Franc et al., 2018; Mark, Toland, Rosenkrantz, Brown, \& Hong, 2018; Spector, Carey, \& Steinberg, 1996), congruent with the enhancing and arousing effects of cybersex (Beutel et al., 2017; Reid, Li, Gilliland, Stein, \& Fong, 2011). A recent study found weaker correlations between cybersex coping motives and sexual desire, and no association between solitary sexual desire and cybersex social motives (Franc et al., 2018).

The aim of this study was therefore to assess the influence of cybersex-related motives and sexual desire on compulsive cybersex use in men and women.

\section{METHODS}

\section{Procedure}

The study was conducted online by posting ads on sexually related sites and forums with the agreement of the webmasters. It was aimed at anyone aged 18 years or over participating in online sexual activity. No payment was made for participation in the study.

\section{Participants}

Following the recruitment procedure, 761 people clicked on the link and 605 agreed to participate in the study. The completion rate progressively decreased, and 358 of the 605 subjects who began the questionnaire continued past the demographic data section. After missing values were removed, the final sample consisted of 306 subjects, i.e., 150 men (49\%) and 156 women (51\%). The age range was $18-69$ years, average $32.63( \pm 10.83)$ years. The average age of males was $33.44( \pm 11.84)$ years and females $31.86( \pm 9.73)$ years. Participants were mainly from Switzerland $(68 \%)$, France (25\%), Belgium (2\%), Canada (1\%), USA (1\%), and other countries. About $73 \%$ of participants were in a relationship. People with a heterosexual orientation represented about $84 \%$ of the sample, those with a bisexual orientation about $12 \%$, and about $4 \%$ stated that they were homosexual.

\section{Measures}

The first part of the questionnaire included questions that explored the participants' sociodemographic characteristics. The remainder of the questionnaire included three tools: (a) the Compulsive Internet Use Scale (CIUS), (b) the Cybersex Motives Questionnaire (CMQ), and (c) the Sexual Desire Inventory-2 (SDI-2).

The CIUS was designed to evaluate addictive Internet use (Meerkerk, Van Den Eijnden, Vermulst, \& Garretsen, 2009) and has been validated as such. It consists of 14 items with a 5-point Likert scale from 0 (never) to 56 (very often). The CIUS has constantly been found to have a unified structure in various studies and samples (Khazaal et al., 2011, 2012; Meerkerk et al., 2009; Wartberg, Petersen,
Kammerl, Rosenkranz, \& Thomasius, 2014). High scores indicate respondents' inability to restrict their use of the Internet. As reported in other studies assessing Internetrelated addictive behaviors (Khazaal et al., 2015), the CIUS has been adapted to assess compulsive cybersex use.

To ensure that the CIUS targeted only cybersex behaviors, we drew the participants' attention to the fact that the word Internet in the scale referred solely to cybersex. The CIUS and other instruments designed to assess Internet addiction have been successfully adapted to study gaming and gambling (Khazaal et al., 2015), and cybersex (Downing, Antebi, \& Schrimshaw, 2014; Varfi et al., 2019; Wéry et al., 2018), without modifying their psychometric properties.

The CIUS items target the typical symptoms of compulsive Internet use mentioned in the literature, such as loss of control, preoccupation, withdrawal symptoms, coping, and mood changes. It has good stability with good internal consistency (Cronbach's $\alpha=.90$; Meerkerk et al., 2009).

The CMQ identifies the motives associated with the use of sexual sites (Franc et al., 2018). It comprises 14 items rated on a 5-point Likert scale from never to always or almost always, structured around three factors: Enhancement, Social, and Coping. The enhancement motive corresponds to the excitement and enjoyment of sexual activity online (i.e., "Because it is exciting" and "Because I like the feeling"). The social motive refers to the affiliation that can be felt by the user through the virtual world that encourages and accepts one's deepest fantasies (i.e., "To be sociable and liked by others" and "To meet somebody"). The third motive refers to coping strategies that reflect the use of cybersex as a way of escaping from reality and disengaging from real-life concerns (i.e., "It comforts me when I'm in a bad mood" and "In order to forget my problems or worries"). The CMQ has satisfactory psychometric qualities. Cronbach's $\alpha$ is .84 for the first factor, .73 for the second factor, and .79 for the third factor (Franc et al., 2018), which is acceptable.

The SDI-2 is used to assess sexual desire, defined as interest in sexual behavior (Spector et al., 1996). It is one of the most frequently used instruments for the assessment of sexual desire (Mark et al., 2018). It consists of 14 items measuring the intensity and importance of desire on a 9-point Likert scale ranging from no desire to strong desire or from not at all important to extremely important. Items measuring the frequency of sexual activities are rated on an 8-point Likert scale ranging from never to more than once a day. The items can be summed to produce an overall score or to produce scores for its two component dimensions: (a) dyadic sexual desire (desire to engage in sexual activity with a partner) and (b) solitary sexual desire (desire to engage in solo sexual activity). Dyadic sexual desire corresponds to Items 1-9, with a total score ranging from 0 to 62 , and solitary sexual desire to the sum of Items 10-13, with a total score ranging from 0 to 23 . Item 14 assesses to what extent participants can do without sexual activity and does not belong to either the solitary or the dyadic desire dimension. Solitary desire concerns the rate of solitary sexual behavior and dyadic desire concerns the frequency of sexual behaviors with a companion (Spector et al., 1996). The SDI-2 has good psychometric qualities with good internal 
consistency of the two factors (.86 and .96, respectively; Spector et al., 1996). Previous studies reported stable psychometric characteristics across samples with different languages and sexual orientations, such as lesbians and gay males (Dosch, Rochat, Ghisletta, Favez, \& Van der Linden, 2016; Mark et al., 2018).

\section{Statistical analysis}

After a descriptive analysis, we used Student's $t$-test to provide gender comparisons and Pearson's correlation analyses to assess links between variables. Where data were missing, scores were replaced by the average of the scores obtained by the individual for the items of the subscale, or for the total score if the questionnaire had no subscale (person-mean imputation). Respondents with a rate of missing responses exceeding $10 \%$ were excluded.

Structural equation modeling (SEM) was performed using the maximum likelihood estimation. Fits were considered to be good if the values of the comparative fit index (CFI) were close to .90 , the $\chi^{2} / d f$ ratio close to 2 , and the root mean square error of approximation (RMSEA) $<0.08$ (Arbuckle \& Wothke, 2003; Byrne, 2010; Hu \& Bentler, 1999). Statistical analyses were performed using software TIBCO Statistica ${ }^{\mathrm{TM}}$ 13.3.0 (TIBCO Software Inc., Palo Alto, CA, USA) and IBM $^{\circledR}$ SPSS $^{\circledR} \operatorname{Amos}^{\mathrm{TM}} 23.00$ (IBM SPSS Software Inc., Wexford, PA, USA).

\section{Ethics}

The study was carried out in accordance with the Declaration of Helsinki. The ethical committee of the Geneva University Hospitals approved the study protocol. Participants were given a full description of the study aims and methods online. They were then asked to give their informed consent online, which allowed them to answer the questionnaires anonymously via SurveyMonkey.

\section{RESULTS}

Descriptive results are presented in Table 1. All distributions can be considered normal. Table 1 also provides the Cronbach's $\alpha$ coefficients used to test the reliability of the scales. These are considered satisfactory when the $\alpha$ coefficient is $>.70$, which was the case here. Table 2 shows the differences in means by gender. Men scored higher than women for cybersex enhancement motives, and also for dyadic and solitary sexual desire (with large effect sizes). When single participants were compared with those living with a partner, there was a significant difference for dyadic sexual desire (41.64 vs. $46.23, t=-2.73, p<.01$, with a medium effect size). The comparison by sexual orientation did not reveal any significant differences, although there was a tendency for bisexual participants to report more sexual desire and to have higher CIUS scores.

Because we found no difference in CIUS scores between men and women, we explored how they were distributed according to the intensity of cybersex use (low, moderate, and high). The ranking of all participants in the three groups (by tertiles) showed that women were mostly in the low and the high compulsive use groups, whereas men were mainly in the moderate-use group (Table 3).

Table 4 presents correlations between CIUS scores and CMQ and SDI-2 subscores. The results highlight significant and positive correlations with all of the subscales. The strongest relationships were observed between the CIUS score and scores for the CMQ coping motives $(r=.52$, $p<.001)$ and the CMQ social motives $(r=.39, p<.001)$, and to a lesser extent between the CIUS score and the enhancement motives score $(r=.28, p<.001)$.

Table 5 presents the correlations by gender. For women, there was a significant relationship between the CIUS score and scores for the CMQ coping motives $(r=.51, p<.001)$ and the CMQ social motives $(r=.49, p<.001)$, and to a lesser extent between the CIUS score and the CMQ enhancement motives score $(r=.34, p<.001)$. Notably, no statistically significant correlations were found between the CIUS score and the sexual desire subscales.

For men, we observed a significant relationship between the CIUS score and the CMQ coping motives score $(r=.54$, $p<.001)$, and also, albeit to a lesser extent, between the CIUS score and scores for the other CMQ motive subscales. In contrast to women, we found an association between the men's CIUS scores and both solitary $(r=.28, p<.001)$ and dyadic sexual desire $(r=.25, p<.001)$.

Finally, we conducted SEM to investigate the relationships between the measured variables (CIUS, CMQ, and SDI-2) and their interdependences (Figure 1). The values of the fits are acceptable $\left(\chi^{2} / d f=3.01, \mathrm{CFI}=0.80\right.$, and RMSEA $=0.08)$. We proceeded in a similar way for men and women separately (see Figures 2 and 3). The CFI value for men was low (0.74). Figure 1 shows the association between the CIUS scores and the CMQ coping motives and

Table 1. Descriptive analyses of CIUS, CMQ, and SDI-2

\begin{tabular}{lccccccccc}
\hline & Mean [95\% CI] & Median & $S D$ & Range & Skewness & Kurtosis & $d($ K-S $)$ & Lilliefors $(p)$ & Cronbach's $\alpha$ \\
\hline CIUS & $19.54[18.16-20.91]$ & 19 & 12.20 & $0-51$ & 0.19 & -0.92 & 0.08 & $<.01$ & .93 \\
CMQ & $23.85[23.04-24.66]$ & 25 & 12.20 & $8-40$ & -0.36 & -0.23 & 0.08 & $<.01$ & .88 \\
$\quad$ enhancement & & & & & & & & & \\
CMQ social & $10.33[9.91-10.74]$ & 11 & 12.20 & $4-20$ & -0.15 & -0.66 & 0.10 & $<.01$ & .72 \\
CMQ coping & $12.70[12.15-13.25]$ & 13 & 12.20 & $5-25$ & 0.06 & -0.85 & 0.08 & $<.01$ & .81 \\
SDI-2 & $64.25[61.96-66.54]$ & 67 & 20.34 & $0-109$ & -0.62 & 0.39 & 0.07 & $<.01$ & .91 \\
SDI-2 dyadic & $44.97[43.48-46.47]$ & 47 & 12.20 & $0-70$ & -0.88 & 1.07 & 0.08 & $<.01$ & .87 \\
SDI-2 solitary & $15.60[14.61-16.59]$ & 17 & 12.20 & $0-31$ & -0.32 & -0.89 & 0.10 & $<.01$ & .93 \\
\hline
\end{tabular}

Note. CIUS: Compulsive Internet Use Scale; CMQ: Cybersex Motives Questionnaire; SDI-2: Sexual Desire Inventory-2; CI: confidence interval; $S D$ : standard deviation; $d(\mathrm{~K}-\mathrm{S})$ : Kolmogorov-Smirnov test. 
Gender differences in cybersex addiction contributors

Table 2. Comparisons of CIUS, CMQ, and SDI-2 scores by gender

\begin{tabular}{|c|c|c|c|c|c|}
\hline & Men & Women & Effect size & & \\
\hline Measure & Mean $(S D)$ & Mean $(S D)$ & (Cohen's $d$ ) & $t$ & $p$ \\
\hline CIUS & $19.30(11.18)$ & $19.76(13.14)$ & -0.04 & -0.33 & .740 \\
\hline CMQ enhancement & $26.25(6.66)$ & $21.55(7.01)$ & 0.65 & 6.01 & .001 \\
\hline CMQ social & $10.18(3.47)$ & $10.47(3.90)$ & -0.08 & -0.67 & .510 \\
\hline CMQ coping & $12.82(4.81)$ & $12.59(4.93)$ & 0.05 & 0.40 & .690 \\
\hline SDI-2 & 71.89 (17.88) & $56.90(19.90)$ & 0.74 & 6.92 & .001 \\
\hline SDI-2 dyadic & $48.35(12.30)$ & $41.73(13.37)$ & 0.50 & 4.51 & .001 \\
\hline SDI-2 solitary & $19.02(7.64)$ & $12.31(8.64)$ & 0.76 & 7.18 & .001 \\
\hline
\end{tabular}

Note. CIUS: Compulsive Internet Use Scale; CMQ: Cybersex Motives Questionnaire; SDI-2: Sexual Desire Inventory-2; SD: standard deviation.

Table 3. Characteristics of the three groups of cybersex users with low, moderate, and high CIUS scores

\begin{tabular}{lccc}
\hline & Low CIUS score $(n=105)$ & Medium CIUS score $(n=102)$ & High CIUS score $(n=99)$ \\
\hline CIUS [mean $(S D)]$ & $6.05(3.84)$ & $19.48(4.05)$ & $33.89(5.28)$ \\
CIUS (range) & $0-12$ & $13-26$ & $27-51$ \\
Age [mean $(S D)]$ & $32.68(11.17)$ & $33.15(11.90)$ & $32.06(9.27)$ \\
In a relationship & $67.62 \%(n=71)$ & $75.49 \%(n=77)$ & $74.75 \%(n=74)$ \\
Men & $46.67 \%(n=49)$ & $56.87 \%(n=58)$ & $43.43 \%(n=43)$ \\
Women & $53.33 \%(n=56)$ & $43.14 \%(n=44)$ & $56.57 \%(n=56)$ \\
Heterosexual orientation & $86.67 \%(n=91)$ & $84.31 \%(n=86)$ & $79.80 \%(n=79)$ \\
Homosexual orientation & $3.81 \%(n=4)$ & $6.86 \%(n=7)$ & $3.03 \%(n=3)$ \\
Bisexual orientation & $9.52 \%(n=10)$ & $8.82 \%(n=9)$ & $17.17 \%(n=17)$ \\
\hline
\end{tabular}

Note. CIUS: Compulsive Internet Use Scale; $S D$ : standard deviation.

Table 4. Correlations between CIUS, CMQ, and SDI-2 scores

\begin{tabular}{|c|c|c|c|c|c|c|c|}
\hline & CIUS & CMQ enhancement & CMQ social & CMQ coping & SDI-2 & SDI-2 dyadic & SDI-2 solitary \\
\hline CIUS & 1 & $.28 * * *$ & $.39 * * *$ & $.52 * * *$ & $.16^{* *}$ & $.15^{* *}$ & $.14 *$ \\
\hline CMQ enhancement & & 1 & $.28 * * *$ & $.55^{* * *}$ & $.56 * * *$ & $.44 * * *$ & $.55 * * *$ \\
\hline CMQ social & & & 1 & $.58 * * *$ & $.16^{* *}$ & $.17^{* *}$ & .10 \\
\hline CMQ coping & & & & 1 & $.22 * * *$ & $.20 * * *$ & $.19^{* *}$ \\
\hline SDI-2 & & & & & 1 & $.91 * * *$ & $.79 * * *$ \\
\hline SDI-2 dyadic & & & & & & 1 & $.48 * *$ \\
\hline SDI-2 solitary & & & & & & & 1 \\
\hline
\end{tabular}

Note. CIUS: Compulsive Internet Use Scale; CMQ: Cybersex Motives Questionnaire; SDI-2: Sexual Desire Inventory-2.

${ }^{*} p<.05 .{ }^{*} p<.01 . * * * p<.001$.

Table 5. Correlations between CIUS, CMQ, and SDI-2 scores in men and women

\begin{tabular}{lccccccc}
\hline Men/women & CIUS & CMQ enhancement & CMQ social & CMQ coping & SDI-2 & SDI-2 dyadic & SDI-2 solitary \\
\hline CIUS & & $.26^{* * *}$ & $.25^{* * *}$ & $.54^{* * *}$ & $.30^{* * *}$ & $.25^{* * *}$ & $.28^{* * *}$ \\
CMQ enhancement & $.34^{* * *}$ & & .08 & $.47^{* * *}$ & $.44^{* * *}$ & $.29^{* * *}$ & $.49^{* * *}$ \\
CMQ social & $.49^{* * *}$ & $.50^{* * *}$ & & $.41^{* * *}$ & .05 & .09 & -.03 \\
CMQ coping & $.51^{* * *}$ & $.65^{* * *}$ & $.74^{* * *}$ & & .15 & .09 & $.18^{* *}$ \\
SDI-2 & .09 & $.55^{* * *}$ & $.29^{* * *}$ & $.30^{* * *}$ & & $.91^{* * *}$ & $.76^{* * *}$ \\
SDI-2 dyadic & .09 & $.48^{* * *}$ & $.27^{* *}$ & $.29^{* * *}$ & $.91^{* * *}$ & $.42^{* * *}$ \\
SDI-2 solitary & .07 & $.48^{* * *}$ & $.23^{* * *}$ & $.21^{* * *}$ & $.76^{* * *}$ & $.44^{* * *}$ & \\
\hline
\end{tabular}

Note. The lower part of the diagonal refers to correlations for men and the upper part for women. CIUS: Compulsive Internet Use Scale; CMQ: Cybersex Motives Questionnaire; SDI-2: Sexual Desire Inventory-2.

${ }^{* *} p<0.01 .{ }^{* * *} p<.001$. 


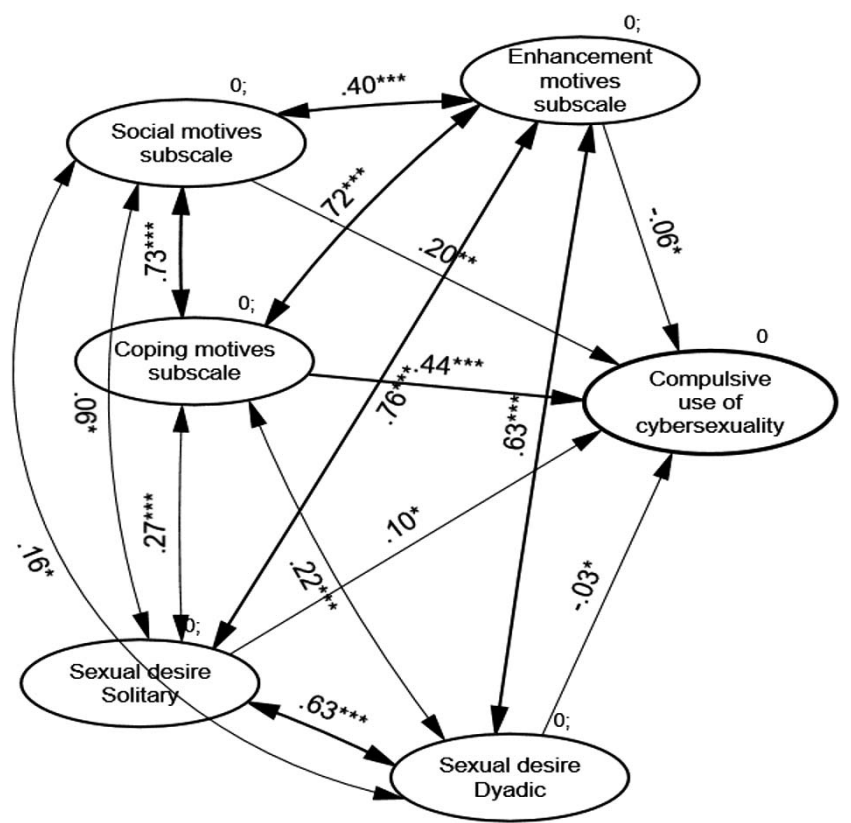

Figure 1. Relationships between the compulsive use of cybersex (Compulsive Internet Use Scale score), cybersex motives (Cybersex Motives Questionnaire subscales), and sexual desire (Sexual Desire Inventory-2 subscales) for the whole sample (men and women). ${ }^{*} p<.05 .{ }^{* *} p<.01 .{ }^{* * *} p<.001 . \chi^{2}=2,295.60$, $d f=764, \chi^{2} / d f=3.01, p<.001, \mathrm{CFI}=0.80$, and RMSEA $=0.08$ [0.08-0.09]. For readability, only latent variables are presented

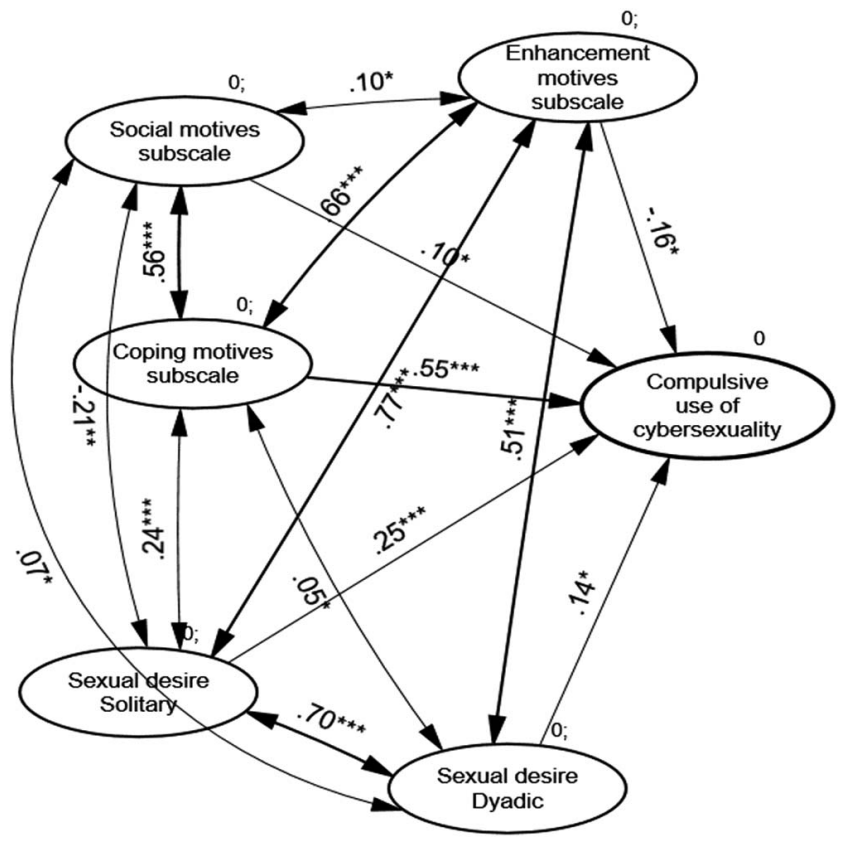

Figure 2. Relationships between compulsive use of cybersex (Compulsive Internet Use Scale score), cybersex motives

(Cybersex Motives Questionnaire subscales), and sexual desire (Sexual Desire Inventory-2 subscales) for men. ${ }^{*} p<.05$. $*^{*} p<.01 . * * * p<.001 . \chi^{2}=1,617.37, d f=764, \chi^{2} / d f=2.12$, $p<.001, \mathrm{CFI}=0.74$, and RMSEA $=0.09$ [0.08-0.09]. For readability, only latent variables are presented

CMQ social motives. It also highlights the importance of the links between CMQ enhancement motives and SDI-2 sexual desire. For men, Figure 2 shows the association between the

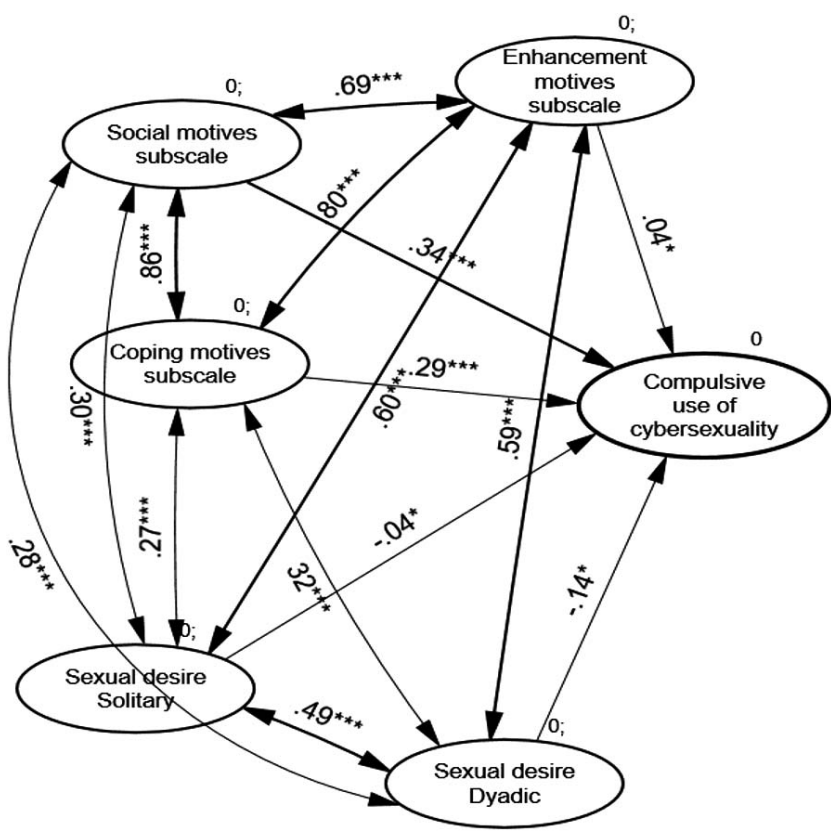

Figure 3. Relationships between compulsive use of cybersex (Compulsive Internet Use Scale score), cybersex motives (Cybersex Motives Questionnaire subscales), and sexual desire (Sexual Desire Inventory-2 subscales) for women. ${ }^{*} p<.05$. ${ }^{* *} p<.01 .{ }^{* * *} p<.001 . \chi^{2}=1,650.29, d f=766, \chi^{2} / d f=2.15$, $p<.001, \mathrm{CFI}=0.80$, and RMSEA $=0.09$ [0.08-0.09]. For readability, only latent variables are presented

CMQ coping motives and the CIUS, with links to SDI-2 sexual desire. Figure 3 for women highlights the association of CMQ social and coping motives with the CIUS.

\section{DISCUSSION}

The results of the study did not reveal significant differences between men and women in the CIUS scores, but they did show that the women participants were mostly in either the low-use or the high-use group. The lack of gender difference is not consistent with previous work (e.g., Kafka, 2010). The presence of subgroups of women in the high-risk group is, however, consistent with other studies on behavioral addictions (Khazaal et al., 2017), showing that subsamples of women are possibly at increased risk of behavioral addictions.

Because we recruited participants through sex-related websites and forums, the study was possibly subject to a self-selection bias (Khazaal et al., 2014). Overinclusion of people with a high level of cybersex use cannot be excluded. Epidemiological conclusions thus cannot be drawn from the study. Nevertheless, the results highlight an association between a number of variables and the CIUS scores of men and women in this sample.

The results suggest the role of CMQ coping, in both genders, and to some extent social motives, especially for women, in compulsive cybersex. The CMQ enhancement motives showed a small association with the CIUS scores, especially for men.

Coping motives refer to escape strategies that the individual sets up in the face of aversive situations (e.g., Barrault et al., 2016; Miner, Coleman, Center, Ross, \& Rosser, 2007). 
They may help people find transient relief during painful moments (Coleman-Kennedy \& Pendley, 2002; Leiblum, 1997). The study results suggest that such motives are associated with compulsive cybersex in both men and women. This finding is consistent with other studies on substance use (Blevins, Banes, Stephens, Walker, \& Roffman, 2016; Grazioli et al., 2018) and other behavioral addictions (Brand et al., 2014; Clarke et al., 2007; Khazaal et al., 2018), as well as with studies showing interactions between negative affect and impulsivity in compulsive cybersex (Wéry et al., 2018). In this study, the CMQ coping motives also had the strongest impact on the CIUS in both genders. At a clinical level, these results suggest the importance of interventions that focus on emotional regulation to help people with compulsive cybersex. Further studies may benefit from including coping skills and cognitive expectancies as possible mediators between the compulsive use of cybersex and coping motives (Brand et al., 2014; Laier, Wegmann, \& Brand, 2018).

As in other studies on behavioral addictions (Müller et al., 2017) and addictive cybersex (Weinstein, Zolek, Babkin, Cohen, \& Lejoyeux, 2015), the study showed some important gender differences. More specifically, social motives were more strongly associated with CIUS scores for women than for men. This is consistent with other studies showing the higher involvement of women in social networks (Dufour et al., 2016). In addition, sexual desire was not found to be associated with CIUS scores in women, whereas an association, albeit small, was found for men.

Although the strongest association between the CIUS score and the other variables was most importantly shown in both genders for the CMQ coping motives, a smaller association was observed for sexual desire among men and for social motives among women. These findings are possibly due to dissimilarities between women and men in sexual desire (Carvalho \& Nobre, 2011). The finding that sexual desire was only a factor in the male participants' compulsive use of cybersex may be linked to gender differences in the way relational and social dimensions of sexuality act on sexual desire (Carvalho \& Nobre, 2011).

In addition to such gender differences, our results suggest that sexual desire plays only a small role (in men), or even no role (in women) in compulsive cybersex use. Furthermore, the CMQ enhancement subscale does not seem to contribute to the CIUS score. This suggests that cybersex addiction is not driven by sex or only to a small extent in men. This finding is consistent with other studies showing that liking sexually explicit videos (Voon et al., 2014) and sexual activities (i.e., number of sexual contacts, satisfaction with sexual contacts, and use of interactive cybersex) is not associated with compulsive cybersex (Laier et al., 2014; Laier, Pekal, \& Brand, 2015). As suggested in other studies on addictive behaviors, the "liking" dimension (hedonic drive) seems to play a smaller role than the "wanting" (incentive salience) and "learning" dimensions (predictive associations and cognitions, e.g., learning about negative emotion relief when using cybersex; Berridge, Robinson, \& Aldridge, 2009; Robinson \& Berridge, 2008).

At first glance, the small role of sexual desire and enhancement motives in compulsive cybersex seems counterintuitive. It appears that the sexual nature of the gratification is not a major drive of the behavior. This observation could be explained by the fact that the CIUS is not a measure of sexual activity or of cybersex use, but an assessment of compulsive cybersex use. The findings are consistent with the process related to maintenance of addictive behaviors. It has been postulated that addictions are maintained by a shift from gratification (i.e., looking for direct sexual rewards) to compensation (i.e., looking for escape from negative moods; Young \& Brand, 2017). To further investigate this question, future studies should include concomitant assessments of cybersex use, sexual behaviors, and compulsive cybersex, together with the nature of the rewards obtained during cybersex use. Ecological momentary assessment could be used to explore these questions (Benarous et al., 2016; Ferreri, Bourla, Mouchabac, \& Karila, 2018; Jones, Tiplady, Houben, Nederkoorn, \& Field, 2018).

This work has several limitations, mainly related to the cross-sectional design, use of self-assessment questionnaires, self-selection biases, and the convenience sample size. The results should be confirmed by future studies, possibly based on the present results, including power analyses and sample size planning as well as detailed assessments of cybersex use (i.e., porn, dating, webcam, and chat) or focusing on a specific activity. The CIUS adapted for cybersex in this study showed good internal consistency (Cronbach's $\alpha=.93$ ). It is a measure of compulsive sexuality, but not an assessment of cybersex use itself, and specific sexual activities were not reported. Further studies should include a description of such activities, perceived rewards linked to cybersex use, as well as an assessment of non-Internet based sexual involvement (i.e., sexual intercourse, etc.) and emotional relationship with partner.

Further psychological variables, such as self-esteem, mood (Park, Hong, Park, Ha, \& Yoo, 2012), impulsivity (Wéry et al., 2018), loneliness (Khazaal et al., 2017; Yong, Inoue, \& Kawakami, 2017), attachment (Favez, Tissot, Ghisletta, Golay, \& Cairo Notari, 2016), and psychiatric comorbidities (Starcevic \& Khazaal, 2017), may play an important role in compulsive cybersex, in addition to emotional and sexual satisfaction in real life.

Funding sources: None.

Authors' contribution: YK, FB-D, and SR contributed in study concept and design. FBB, RC, SR, and YK contributed in statistical analysis and interpretation of data. YK contributed in recruitment. FBB, SR, FB-D, RC, and YK contributed in drafting of the manuscript.

\section{Conflict of interest: None.}

Acknowledgements: The authors would like to thank Barbara Every, ELS, of BioMedical Editor, and Elizabeth Yates for English language editing. They would also like to thank the study participants. 


\section{REFERENCES}

Albright, J. M. (2008). Sex in America online: An exploration of sex, marital status, and sexual identity in Internet sex seeking and its impacts. The Journal of Sex Research, 45(2), 175-186. doi:10.1080/00224490801987481

Allen, A., Kannis-Dymand, L., \& Katsikitis, M. (2017). Problematic Internet pornography use: The role of craving, desire thinking, and metacognition. Addictive Behaviors, 70, 65-71. doi:10.1016/ j.addbeh.2017.02.001

Arbuckle, J. L., \& Wothke, W. (2003). AMOS (version 5.0) [Computer software]. Chicago, IL: SmallWaters Corporation.

Barrault, S., Hegbe, K., Bertsch, I., \& Courtois, R. (2016). Relation entre les événements de vie traumatiques de l'enfance, le trouble de personnalité borderline et les conduites cybersexuelles problématiques [Relationship between traumatic life events of childhood, borderline personality disorder and problematic cybersexual behavior]. Psychotropes, 22(3), 65-81. doi:10.3917/psyt. 223.006510.3917/psyt.223.0065

Benarous, X., Edel, Y., Consoli, A., Brunelle, J., Etter, J.-F., Cohen, D., \& Khazaal, Y. (2016). Ecological momentary assessment and smartphone application intervention in adolescents with substance use and comorbid severe psychiatric disorders: Study protocol. Frontiers in Psychiatry, 7, 157. doi:10.3389/fpsyt.2016.00157

Benschop, A., Liebregts, N., van der Pol, P., Schaap, R., Buisman, R., van Laar, M., van den Brink, W., de Graaf, R., \& Korf, D. J. (2015). Reliability and validity of the Marijuana Motives Measure among young adult frequent cannabis users and associations with cannabis dependence. Addictive Behaviors, 40, 91-95. doi:10.1016/j.addbeh.2014.09.003

Berridge, K. C., Robinson, T. E., \& Aldridge, J. W. (2009). Dissecting components of reward: 'Liking', 'wanting', and learning. Current Opinion in Pharmacology, 9(1), 65-73. doi:10.1016/j.coph.2008.12.014

Beutel, M. E., Giralt, S., Wölfling, K., Stöbel-Richter, Y., SubicWrana, C., Reiner, I., Tibubos, A. N., \& Brähler, E. (2017). Prevalence and determinants of online-sex use in the German population. PLoS One, 12(6), e0176449. doi:10.1371/journal. pone.0176449

Billieux, J., Chanal, J., Khazaal, Y., Rochat, L., Gay, P., Zullino, D., \& Van der Linden, M. (2011). Psychological predictors of problematic involvement in massively multiplayer online roleplaying games: Illustration in a sample of male cybercafé players. Psychopathology, 44(3), 165-171. doi:10.1159/000322525

Blevins, C. E., Banes, K. E., Stephens, R. S., Walker, D. D., \& Roffman, R. A. (2016). Motives for marijuana use among heavy-using high school students: An analysis of structure and utility of the Comprehensive Marijuana Motives Questionnaire. Addictive Behaviors, 57, 42-47. doi:10.1016/j. addbeh.2016.02.005

Brand, M., Laier, C., Pawlikowski, M., Schächtle, U., Schöler, T., \& Altstötter-Gleich, C. (2011). Watching pornographic pictures on the Internet: Role of sexual arousal ratings and psychological-psychiatric symptoms for using Internet sex sites excessively. Cyberpsychology, Behavior, and Social Networking, 14(6), 371-377. doi:10.1089/cyber.2010.0222

Brand, M., Laier, C., \& Young, K. S. (2014). Internet addiction: Coping styles, expectancies, and treatment implications. Frontiers in Psychology, 5, 1256. doi:10.3389/fpsyg.2014. 01256
Byrne, B. M. (2010). Structural equation modeling with Amos: Basic concepts, applications, and programming (2nd ed.). New York, NY: Routledge.

Carvalho, J., \& Nobre, P. (2011). Gender differences in sexual desire: How do emotional and relationship factors determine sexual desire according to gender? Sexologies, 20(4), 207-211. doi:10.1016/j.sexol.2011.08.010

Clarke, D., Tse, S., Abbott, M. W., Townsend, S., Kingi, P., \& Manaia, W. (2007). Reasons for starting and continuing gambling in a mixed ethnic community sample of pathological and non-problem gamblers. International Gambling Studies, 7(3), 299-313. doi:10.1080/14459790701601455

Coleman-Kennedy, C., \& Pendley, A. (2002). Assessment and diagnosis of sexual addiction. Journal of the American Psychiatric Nurses Association, 8(5), 143-151. doi:10.1067/ mpn.2002.128827

Commission of the European Communities. (2002). eEurope 2002: Quality criteria for health related websites. Journal of Medical Internet Research, 4(3), E15. doi:10.2196/ jmir.4.3.e15

Döring, N. M. (2009). The Internet's impact on sexuality: A critical review of 15 years of research. Computers in Human Behavior, 25(5), 1089-1101. doi:10.1016/j.chb.2009.04.003

Dosch, A., Rochat, L., Ghisletta, P., Favez, N., \& Van der Linden, M. (2016). Psychological factors involved in sexual desire, sexual activity, and sexual satisfaction: A multi-factorial perspective. Archives of Sexual Behavior, 45(8), 2029-2045. doi:10.1007/s10508-014-0467-z

Downing, M. J., Antebi, N., \& Schrimshaw, E. W. (2014). Compulsive use of Internet-based sexually explicit media: Adaptation and validation of the Compulsive Internet Use Scale (CIUS). Addictive Behaviors, 39(6), 1126-1130. doi:10.1016/j.addbeh.2014.03.007

Dufour, M., Brunelle, N., Tremblay, J., Leclerc, D., Cousineau, M.-M., Khazaal, Y., Légaré, A. A., Rousseau, M., \& Berbiche, D. (2016). Gender difference in Internet use and Internet problems among Quebec high school students. The Canadian Journal of Psychiatry, 61(10), 663-668. doi:10.1177/ 0706743716640755

Favez, N., Tissot, H., Ghisletta, P., Golay, P., \& Cairo Notari, S. (2016). Validation of the French version of the Experiences in Close Relationships-Revised (ECR-R) Adult Romantic Attachment Questionnaire. Swiss Journal of Psychology, 75(3), 113-121. doi:10.1024/1421-0185/a000177

Ferreri, F., Bourla, A., Mouchabac, S., \& Karila, L. (2018). e-Addictology: An overview of new technologies for assessing and intervening in addictive behaviors. Frontiers in Psychiatry, 9, 51. doi:10.3389/fpsyt.2018.00051

Franc, E., Khazaal, Y., Jasiowka, K., Lepers, T., BianchiDemicheli, F., \& Rothen, S. (2018). Factor structure of the Cybersex Motives Questionnaire. Journal of Behavioral Addictions, 7(3), 601-609. doi:10.1556/2006.7.2018.67

Frangos, C. C., Frangos, C. C., \& Sotiropoulos, I. (2010). Problematic Internet use among Greek university students: An ordinal logistic regression with risk factors of negative psychological beliefs, pornographic sites, and online games. Cyberpsychology, Behavior, and Social Networking, 14(1-2), 51-58. doi:10.1089/cyber.2009.0306

Grazioli, V. S., Bagge, C. L., Studer, J., Bertholet, N., RougemontBücking, A., Mohler-Kuo, M., Daeppen, J. B., \& Gmel, G. (2018). Depressive symptoms, alcohol use and coping drinking 
motives: Examining various pathways to suicide attempts among young men. Journal of Affective Disorders, 232, 243-251. doi:10.1016/j.jad.2018.02.028

Grov, C., Gillespie, B. J., Royce, T., \& Lever, J. (2011). Perceived consequences of casual online sexual activities on heterosexual relationships: A U. S. online survey. Archives of Sexual Behavior, 40(2), 429-439. doi:10.1007/s10508-010-9598-Z

Grubbs, J. B., Volk, F., Exline, J. J., \& Pargament, K. I. (2015). Internet pornography use: Perceived addiction, psychological distress, and the validation of a brief measure. Journal of Sex and Marital Therapy, 41(1), 83-106. doi:10.1080/0092623X. 2013.842192

Hu, L., \& Bentler, P. M. (1999). Cutoff criteria for fit indexes in covariance structure analysis: Conventional criteria versus new alternatives. Structural Equation Modeling: A Multidisciplinary Journal, 6(1), 1-55. doi:10.1080/10705519909540118

Jones, A., Tiplady, B., Houben, K., Nederkoorn, C., \& Field, M. (2018). Do daily fluctuations in inhibitory control predict alcohol consumption? An ecological momentary assessment study. Psychopharmacology, 235(5), 1487-1496. doi:10.1007/ s00213-018-4860-5

Kafka, M. P. (2010). Hypersexual disorder: A proposed diagnosis for DSM-V. Archives of Sexual Behavior, 39(2), 377-400. doi:10.1007/s10508-009-9574-7

Karila, L., Wéry, A., Weinstein, A., Cottencin, O., Petit, A., Reynaud, M., \& Billieux, J. (2014). Sexual addiction or hypersexual disorder: Different terms for the same problem? A review of the literature. Current Pharmaceutical Design, 20(25), 4012-4020. doi:10.2174/13816128113199990619

Khazaal, Y., Achab, S., Billieux, J., Thorens, G., Zullino, D., Dufour, M., \& Rothen, S. (2015). Factor structure of the Internet Addiction Test in online gamers and poker players. JMIR Mental Health, 2(2), e12. doi:10.2196/mental.3805

Khazaal, Y., Breivik, K., Billieux, J., Zullino, D., Thorens, G., Achab, S., Gmel, G., \& Chatton, A. (2018). Game Addiction Scale assessment through a nationally representative sample of young adult men: Item response theory graded-response modeling. Journal of Medical Internet Research, 20(8), e10058. doi:10.2196/10058

Khazaal, Y., Chatton, A., Achab, S., Monney, G., Thorens, G., Dufour, M., Zullino, D., \& Rothen, S. (2017). Internet gamblers differ on social variables: A latent class analysis. Journal of Gambling Studies, 33(3), 881-897. doi:10.1007/ s10899-016-9664-0

Khazaal, Y., Chatton, A., Atwi, K., Zullino, D., Khan, R., \& Billieux, J. (2011). Arabic validation of the Compulsive Internet Use Scale (CIUS). Substance Abuse Treatment, Prevention, and Policy, 6(1), 32. doi:10.1186/1747-597X-6-32

Khazaal, Y., Chatton, A., Horn, A., Achab, S., Thorens, G., Zullino, D., \& Billieux, J. (2012). French validation of the Compulsive Internet Use Scale (CIUS). Psychiatric Quarterly, 83(4), 397-405. doi:10.1007/s11126-012-9210-x

Khazaal, Y., van Singer, M., Chatton, A., Achab, S., Zullino, D., Rothen, S., Khan, R., Billieux, J., \& Thorens, G. (2014). Does self-selection affect samples' representativeness in online surveys? An investigation in online video game research. Journal of Medical Internet Research, 16(7), e164. doi:10.2196/ jmir.2759

Király, O., Urbán, R., Griffiths, M. D., Ágoston, C., Nagygyörgy, K., Kökönyei, G., \& Demetrovics, Z. (2015). The mediating effect of gaming motivation between psychiatric symptoms and problematic online gaming: An online survey. Journal of Medical Internet Research, 17(4), e88. doi:10.2196/jmir.3515

Laier, C., \& Brand, M. (2014). Empirical evidence and theoretical considerations on factors contributing to cybersex addiction from a cognitive-behavioral view. Sexual Addiction \& Compulsivity, 21(4), 305-321. doi:10.1080/10720162.2014.970722

Laier, C., Pawlikowski, M., Pekal, J., Schulte, F. P., \& Brand, M. (2013). Cybersex addiction: Experienced sexual arousal when watching pornography and not real-life sexual contacts makes the difference. Journal of Behavioral Addictions, 2(2), 100-107. doi:10.1556/JBA.2.2013.002

Laier, C., Pekal, J., \& Brand, M. (2014). Cybersex addiction in heterosexual female users of Internet pornography can be explained by gratification hypothesis. Cyberpsychology, Behavior, and Social Networking, 17(8), 505-511. doi:10.1089/cyber.2013.0396

Laier, C., Pekal, J., \& Brand, M. (2015). Sexual excitability and dysfunctional coping determine cybersex addiction in homosexual males. Cyberpsychology, Behavior, and Social Networking, 18(10), 575-580. doi:10.1089/cyber.2015.0152

Laier, C., Wegmann, E., \& Brand, M. (2018). Personality and cognition in gamers: Avoidance expectancies mediate the relationship between maladaptive personality traits and symptoms of Internet-gaming disorder. Frontiers in Psychiatry, 9, 304-304. doi:10.3389/fpsyt.2018.00304

Leiblum, S. R. (1997). Sex and the net: Clinical implications. Journal of Sex Education and Therapy, 22(1), 21-27. doi:10.1080/01614576.1997.11074167

Levine, S. B. (2003). The nature of sexual desire: A clinician's perspective. Archives of Sexual Behavior, 32(3), 279-285. doi:10.1023/A:1023421819465

Mark, K. P., Toland, M. D., Rosenkrantz, D. E., Brown, H. M., \& Hong, S.-H. (2018). Validation of the Sexual Desire Inventory for lesbian, gay, bisexual, trans, and queer adults. Psychology of Sexual Orientation and Gender Diversity, 5(1), 122-128. doi:10.1037/sgd0000260

Meerkerk, G. J., Van Den Eijnden, R. J. J. M., Vermulst, A. A., \& Garretsen, H. F. L. (2009). The Compulsive Internet Use Scale (CIUS): Some psychometric properties. CyberPsychology \& Behavior, 12(1), 1-6. doi:10.1089/cpb.2008.0181

Miner, M. H., Coleman, E., Center, B. A., Ross, M., \& Rosser, B. R. S. (2007). The Compulsive Sexual Behavior Inventory: Psychometric properties. Archives of Sexual Behavior, 36(4), 579-587. doi:10.1007/s10508-006-9127-2

Müller, M., Brand, M., Mies, J., Lachmann, B., Sariyska, R. Y., \& Montag, C. (2017). The 2D:4D marker and different forms of Internet use disorder. Frontiers in Psychiatry, 8, 213. doi:10.3389/fpsyt.2017.00213

Park, S., Hong, K.-E. M., Park, E. J., Ha, K. S., \& Yoo, H. J. (2012). The association between problematic Internet use and depression, suicidal ideation and bipolar disorder symptoms in Korean adolescents. Australian and New Zealand Journal of Psychiatry, 47(2), 153-159. doi:10.1177/000486 7412463613

Pfaus, J. G. (2009). Reviews: Pathways of sexual desire. The Journal of Sexual Medicine, 6(6), 1506-1533. doi:10.1111/j. 1743-6109.2009.01309.x

Philaretou, A. G., Mahfouz, A. Y., \& Allen, K. R. (2005). Use of Internet pornography and men's well-being. International Journal of Men's Health, 4(2), 149-169. doi:10.3149/ jmh.0402.149 
Putnam, D. E., \& Maheu, M. M. (2000). Online sexual addiction and compulsivity: Integrating web resources and behavioral telehealth in treatment. Sexual Addiction \& Compulsivity, 7(1-2), 91-112. doi:10.1080/10720160008400209

Reid, R. C., Li, D. S., Gilliland, R., Stein, J. A., \& Fong, T. (2011). Reliability, validity, and psychometric development of the Pornography Consumption Inventory in a sample of hypersexual men. Journal of Sex and Marital Therapy, 37(5), 359-385. doi:10.1080/0092623X.2011.607047

Robinson, T. E., \& Berridge, K. C. (2008). The incentive sensitization theory of addiction: Some current issues. Philosophical Transactions of the Royal Society B: Biological Sciences, 363(1507), 3137-3146. doi:10.1098/rstb.2008.0093

Southern, S. (2008). Treatment of compulsive cybersex behavior. Psychiatric Clinics, 31(4), 697-712. doi:10.1016/j. psc.2008.06.003

Spector, I. P., Carey, M. P., \& Steinberg, L. (1996). The Sexual Desire Inventory: Development, factor structure, and evidence of reliability. Journal of Sex and Marital Therapy, 22(3), 175-190. doi:10.1080/00926239608414655

Starcevic, V., \& Khazaal, Y. (2017). Relationships between behavioural addictions and psychiatric disorders: What is known and what is yet to be learned? Frontiers in Psychiatry, 8, 53. doi: $10.3389 /$ fpsyt. 2017.00053

Sumter, S. R., Vandenbosch, L., \& Ligtenberg, L. (2017). Love me Tinder: Untangling emerging adults' motivations for using the dating application Tinder. Telematics and Informatics, 34(1), 67-78. doi:10.1016/j.tele.2016.04.009

Varfi, N., Rothen, S., Jasiowka, K., Lepers, T., Bianchi-Demicheli, F., \& Khazaal, Y. (2019). Sexual desire, mood, attachment style, impulsivity, and self-esteem as predictive factors for addictive cybersex. JMIR Mental Health, 6(1), e9978. doi:10.2196/mental.9978

Voon, V., Mole, T. B., Banca, P., Porter, L., Morris, L., Mitchell, S., Lapa, T. R., Karr, J., Harrison, N. A., Potenza, M. N., \& Irvine, M. (2014). Neural correlates of sexual cue reactivity in individuals with and without compulsive sexual behaviours. PLoS One, 9(7), e102419. doi:10.1371/journal.pone.0102419

Wartberg, L., Petersen, K.-U., Kammerl, R., Rosenkranz, M., \& Thomasius, R. (2014). Psychometric validation of a German version of the Compulsive Internet Use Scale. Cyberpsychology, Behavior, and Social Networking, 17(2), 99-103. doi:10.1089/cyber.2012.0689

Weinstein, A. M., Zolek, R., Babkin, A., Cohen, K., \& Lejoyeux, M. (2015). Factors predicting cybersex use and difficulties in forming intimate relationships among male and female users of cybersex. Frontiers in Psychiatry, 6, 54. doi:10.3389/ fpsyt.2015.00054

Wéry, A., \& Billieux, J. (2017). Problematic cybersex: Conceptualization, assessment, and treatment. Addictive Behaviors, 64, 238-246. doi:10.1016/j.addbeh.2015.11.007

Wéry, A., Deleuze, J., Canale, N., \& Billieux, J. (2018). Emotionally laden impulsivity interacts with affect in predicting addictive use of online sexual activity in men. Comprehensive Psychiatry, 80, 192-201. doi:10.1016/j. comppsych.2017.10.004

Wéry, A., Karila, L., Sutter, P. D., \& Billieux, J. (2014). Conceptualisation, évaluation et traitement de la dépendance cybersexuelle: Une revue de la littérature [Conceptualization, assessment, and treatment of cybersexual addiction: A review of the literature]. Canadian Psychology/Psychologie Canadienne, 55(4), 266-281. doi: $10.1037 / \mathrm{a} 0038103$

Yong, R. K. F., Inoue, A., \& Kawakami, N. (2017). The validity and psychometric properties of the Japanese version of the Compulsive Internet Use Scale (CIUS). BMC Psychiatry, 17(1), 201. doi:10.1186/s12888-017-1364-5

Young, K. S. (2008). Internet sex addiction: Risk factors, stages of development, and treatment. American Behavioral Scientist, 52(1), 21-37. doi:10.1177/0002764208321339

Young, K. S., \& Brand, M. (2017). Merging theoretical models and therapy approaches in the context of Internet gaming disorder: A personal perspective. Frontiers in Psychology, 8, 1853. doi:10.3389/fpsyg.2017.01853

Zanetta Dauriat, F., Zermatten, A., Billieux, J., Thorens, G., Bondolfi, G., Zullino, D., \& Khazaal, Y. (2011). Motivations to play specifically predict excessive involvement in massively multiplayer online role-playing games: Evidence from an online survey. European Addiction Research, 17(4), 185-189. doi:10.1159/000326070 